\title{
Nutzung von Kundendaten und -feedback zur Produkt- und Serviceoptimierung
}

\author{
Laura Braun und Sven Reinecke
}

\begin{abstract}
Zusammenfassung
Aufgrund der wachsenden Auswahl diverser Anbieter, Produkte und Dienstleistungen im Markt sehen sich heutige Unternehmen mit steigenden Kundenanforderungen sowie dem Wunsch nach sofortiger und individueller Behandlung konfrontiert. Das systematische Management von Kundendaten sowie die konkrete Erhebung und Nutzung von Feedback bieten nicht nur die Möglichkeit einer effektiven und effizienten Kundenansprache, sondern auch eine Individualisierung und Optimierung eigener Services und Produkte. Der vorliegende Beitrag beschäftigt sich mit den Vorbedingungen, Handlungsoptionen und Effekten einer individualisierten Leistungsgestaltung. Zunächst wird auf die Notwendigkeit der Datenerhebung und -anreicherung als Basis der Nutzung von Kundendaten eingegangen. Im Anschluss werden Möglichkeiten und Gestaltungsmaßnahmen in der Interaktions- und Leistungsoptimierung anhand konkreter Beispiele aufgezeigt. Am Ende des Beitrages werden relevante Erkenntnisse für Entscheidungsträger mit Hilfe von Leitfragen für ein managementorientiertes Vorgehen in der kundendatenzentrierten Produktund Servicegestaltung zusammengefasst.
\end{abstract}

L. Braun $(\bowtie)$

SAP SE Schweiz, Institut für Marketing der Universität St. Gallen, St. Gallen, Schweiz

E-Mail: laura.braun@unisg.ch

S. Reinecke

Institut für Marketing der Universität St. Gallen, St. Gallen, Schweiz

E-Mail: sven.reinecke@ unisg.ch 


\subsection{Steigerung von Effektivität und Effizienz durch eine datenbasierte Service- und Produktgestaltung}

Aufgrund der wachsenden Auswahl an Anbietern, Produkten und Services im Markt sehen sich Unternehmen mit steigenden Kundenanforderungen sowie dem Wunsch nach sofortiger und individueller Behandlung konfrontiert (siehe auch Bughin et al. 2010; Kunz et al. 2017; Lehrer et al. 2018). Neue Technologien und die umfassende Digitalisierung von Kundeninteraktionsprozessen bieten heute die Möglichkeit, das Online- und Offlineverhalten von Kunden abzubilden und auf Basis holistischer Kundenprofile entsprechende Potenziale auszuschöpfen. Hierbei zeigt sich, dass ein systematisches Management vorhandener Kundendaten sowie die gezielte Erhebung und Nutzung von Kundenfeedback nicht nur die Performance der Ansprache erhöht, sondern darüber hinaus eine Individualisierung und Optimierung eigener Services und Produkte eröffnet. Als Auszug erfolgreicher Umsetzungsbeispiele lassen sich hier diverse Unternehmen wie Zalando, Amazon, Nike, Harrods, Modomoto, Ovomaltine oder Swiss International Air Lines anführen.

Eine datenbasierte Outside-In-Perspektive in der Leistungsgestaltung und -optimierung bietet diverse Vorteile im Sinne einer effektiveren und effizienteren Kundenansprache: durch eine Gestaltung relevanter Interaktionsinhalte, -prozesse und -maßnahmen sowie der firmeneigenen Angebotspalette können Kunden adäquater bedient werden. Diverse Studien und Case Studies belegen diese positiven Effekte hinsichtlich der Effektivität von Personalisierungs- und Customization-Maßnahmen und zeigen, dass Kunden nicht nur häufiger von personalisierte Angebote angesprochen werden, sondern auch im Vergleich zu Standardangeboten mit höherer Wahrscheinlichkeit einen Kaufabschluss vornehmen (z. B. Bliemel et al. 2013; Dorotic et al. 2012; Rust und Verhoef 2005).

Weiterhin ermöglicht das Wissen um Kundenverhalten und -präferenzen eine Vermeidung ineffektiver Marketingaktivität, was wiederum Ressourcenersparnisse im Sinne von Effizienz-Steigerung in Aussicht stellt. Entsprechende Effekte der Effektivitäts- und Effizienzsteigerung können im Kern wie folgt zusammengefasst werden (siehe Abb. 22.1):

Im Folgenden sollen Handlungsempfehlungen für die systematische Nutzung von Kundendaten und -feedback zur Service- und Produktoptimierung gegeben werden, um dargestellte Potenziale der Effektivität und Effizienz auszuschöpfen. Dabei sollen nicht nur die Vorteile eines solchen daten- und dadurch kundenzentrierten Vorgehens aufgezeigt, sondern auch relevante Voraussetzungen für ein entsprechendes Management erläutert werden.

\subsection{Datenerhebung und Anreicherung als Basis der Datennutzung}

Die erfolgreiche Integration und Nutzung von Kundendaten und Feedback birgt für Unternehmen zentrale Herausforderungen. Um Informationen über Kundeninteressen, -bedürfnisse und -verhalten sinnvoll in die Produkt- und Servicegestaltung transferieren zu können, bedarf es allgemein einer objektiven, verlässlichen (reliablen) und validen Datenbasis, die sich im Ergebnis nicht nur durch Quantität, sondern besonders durch Qualität auszeich- 


\section{Steigerung der Effektivität}

1. Steigerung der Relevanz der Angebote, höhere Turnover-Rates

2. Stärkung der Kundenbindung

3. Ableitung von Schemata zur Optimierung zukünftiger Interaktionen

4. Generell: Steigerung des Erreichens gesetzter Ziele im Sinne von Kosten, Zeit, Qualität, Flexibilität \& Sicherheit

\section{Steigerung der Effizienz}

1. Weniger Ressourcenaufwand je konvertiertem Kunden (Output/Input Ratio)

2. Weniger Ressourcenaufwand je konvertiertem Kunden im Vergleich zu Standardmassnahmen

3. Möglichkeit der Automatisierung von Prozessen und dadurch gleichzeitige Aufwandsreduktion

Abb. 22.1 Effektivität und Effizienz individualisierter Leistungen. (Quelle: eigene Darstellung)

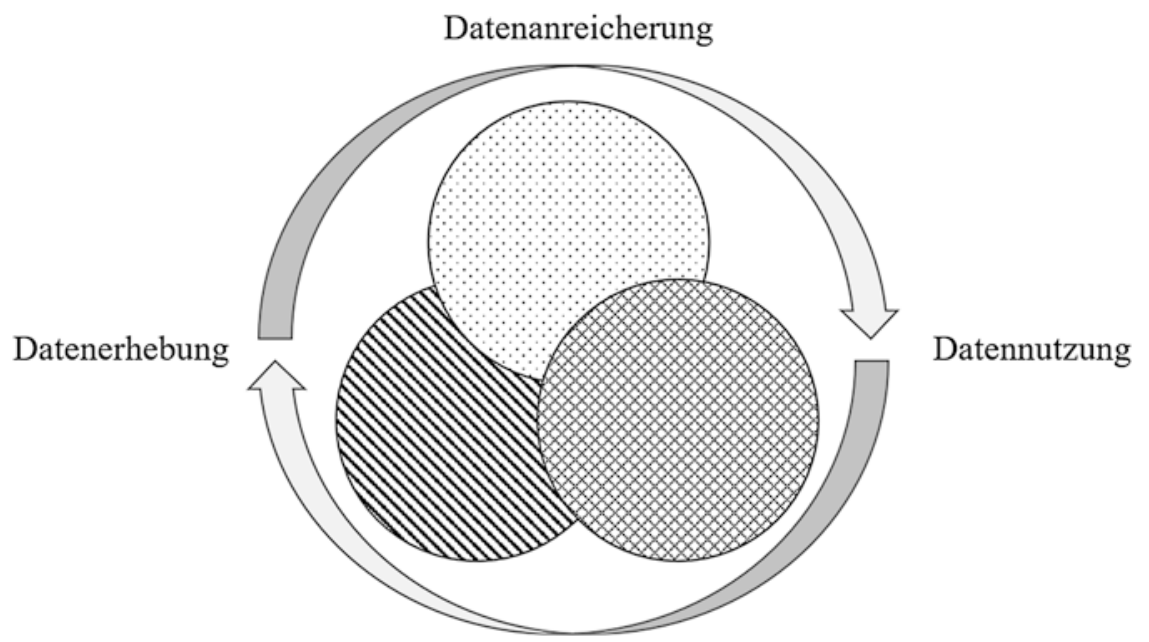

Abb. 22.2 Schematische Darstellung des Zusammenhanges von Datenerhebung, -anreicherung und -nutzung. (Quelle: eigene Darstellung)

net. Unternehmen, die eine kunden- bzw. datenzentrierte Leistungsgestaltung anstreben, müssen sich daher genau mit den Möglichkeiten von Datenerhebung und -anreicherung auseinandersetzen, um die Potenziale, aber auch Grenzen der Nutzung identifizieren zu können. Somit sind Datenerhebung, -anreicherung und -nutzung nicht als getrennte Prozesse, sondern als drei integrierte Aufgaben des Unternehmens anzusehen (siehe Abb. 22.2). 
Beginnend mit Erläuterungen zur Datenerhebung wird in den folgenden Abschnitten (Abschn. 22.2.1, 22.2.2 und 22.2.3) im Detail auf die Möglichkeiten und Herausforderungen dieser Tätigkeiten sowie deren Zusammenspiel eingegangen.

\subsubsection{Datenerhebung}

Ziel der kundenbasierten Produkt- und Serviceoptimierung ist es, Leistungen zu gestalten, die nicht nur die heutigen Kunden, deren Bedürfnisse und Verhaltensweisen bedienen, sondern möglichst auch zukünftiges Interaktionsverhalten zwischen Kunden und Unternehmen adressieren. Aus diesem Grund ist nicht nur die Quantität, sondern auch die Qualität erhobener Daten von besonderer Bedeutung. Als Orientierung zur Einschätzung der Datenqualität können die allgemeinen Gütekriterien der Sozialforschung herangezogen werden (z. B. Wolf und Best 2010). Diese sind Objektivität, Reliabilität und Validität. Folgend werden diese kurz vor dem Hintergrund der aktuellen Thematik skizziert:

\section{Objektivität}

Objektivität beschreibt in diesem Kontext, inwieweit die Daten, die über Kunden und deren Verhalten generiert wurden, unabhängig von jeglichen Einflüssen erhoben, ausgewertet und interpretiert wurden. Das bedeutet, dass erstens die Art der Erhebung bzw. die verantwortliche Person in der Erhebung, zweitens die Auswertungsweise bzw. die auswertende Instanz und drittens die Interpretation der generierten Daten nicht zu unterschiedlichen Erkenntnissen kommen sollten (siehe auch Rammstedt 2010; Rost 1996). Ein Beispiel wäre etwa, dass alle Informationen, die in einem Beratungsgespräch über einen Kunden generiert wurden, gleich sind, unabhängig davon, ob Verkäufer A oder Verkäufer B die Unterhaltung geführt bzw. die Daten erhoben hat.

\section{Reliabilität}

Reliabilität beschreibt die Genauigkeit, mit der ein bestimmtes Merkmal auf Grundlage der Erhebungsmethode erfasst wird, bzw. im Umkehrschluss, wie fehleranfällig die Daten in der Erhebung etwa durch situative Faktoren sind (z. B. Rost 1996). Als Beispiel kann hier die Messung des grundsätzlichen Produktinteresses gewählt werden, deren Ergebnis je nach Situation und Erhebungsmethode sehr anfällig für Verzerrungen und dadurch sehr ungenau sein kann.

\section{Validität}

Die Validität von Informationen wird dadurch bestimmt, inwieweit Daten tatsächlich das messen, was beobachtet werden soll. Da oftmals eine direkte Messung, z. B. von Kundenmeinungen nicht möglich ist, bedient man sich indirekter Erhebungsverfahren, die Aussagen über die zugrunde liegenden Merkmale machen sollen (z. B. Rammstedt 2010). Beispielsweise kann man die Intention für einen Onlinekauf nicht direkt beim Kunden erfragen, jedoch indirekt über Maße, wie die Anzahl an Besuchen der Produkt- 
website, Verweildauer oder Interaktion mit Kundenbewertungen erfassen. Voraussetzungen für die Validität erhobener Daten sind die anderen beiden Gütekriterien Objektivität und Reliabilität.

Widmet man sich nun der konkreten Datenerhebung, so zeigt sich, dass vor allem im Zuge des technologischen Fortschritts die Bandbreite an Datenbezugsquellen rasant wächst (z. B. Bughin et al. 2010). Neben offline generierten Daten (z. B. aus Mitarbeiterprotokollen der direkten Kundeninteraktion im Geschäft) stehen heute online- bzw. sensorgenerierte Informationen zur Verfügung, die für Unternehmen unendliche Potenziale der Leistungsgestaltung ermöglichen (z. B. Kunz et al. 2017). Letztere bieten für Unternehmen den besonderen Vorteil, mit vergleichsweise wenig Aufwand eine große Menge an Daten direkt und in Echtzeit generieren zu können (vgl. auch Braun et al. 2017).

Nimmt man die Online- und Offline-Bezugsquellen zusammen, ergeben sich vielfältige Informationen, die Auskunft über aktuelle Präferenzen und Verhaltensweisen von Kunden geben und damit Grundlage zur Leistungsgestaltung sein können. Hierbei sind zwei Mechanismen zu unterscheiden:

1. Daten, die durch die Unternehmen erhoben bzw. in der Interaktion mit Kunden generiert werden.

2. Daten, welche von Kunden durch Eigeninitiative selbst zur Verfügung gestellt werden.

In Abb. 22.3 werden auszugsweise Beispiele für diese beiden Prozesse vorgestellt:

Um das vollständige Potenzial der Datenerhebung ausschöpfen zu können, ist eine Bereitstellung verschiedenartiger Dialogmöglichkeiten für den Kunden zur Interaktion mit dem Unternehmen förderlich. Besonders im Online-Bereich können Unternehmen von zahlreichen Maßnahmen wie z. B. Kontaktformularen, Online-Umfragen, direkten Hilfefunktionen und Chatmöglichkeiten sowie Feedback-Formularen Gebrauch machen, die effizient Informationen erheben, dokumentieren und auswerten lassen (Bliemel et al. 2000).

Auch wenn sich eine Kundeninteraktion offline im Vergleich deutlich ressourcenintensiver gestaltet, bietet diese die Vorteile, spezifischer auf den Kunden eingehen zu können und dadurch tiefergehende bzw. direkte Informationen erfragen zu können (vgl. auch Braun et al. 2017). Neben der klassischen qualitativen und quantitativen Marktforschung als Informationsquelle können so gezielte Kundengespräche im Verkauf relevante Erkenntnisse generieren, die sogar bei der möglichen Interpretation anderer Daten, wie z. B. Online-Interaktionen, helfen können (siehe auch Reinecke und Wortmann 2018).

Weiterhin bieten Instrumente wie unternehmenseigene oder -übergreifende Loyalitätsprogramme eine direkte Möglichkeit, Informationen von Einzelkunden aus Online- und Offline-Kanälen zu integrieren. Durch ein Programm als Möglichkeit zur Kundenidentifikation an diversen Touchpoints können konkrete Informationen über soziodemographische Merkmale des Kunden mit dem individuellen Such- und Kaufverhalten erhoben und miteinander in Beziehung gebracht werden (siehe auch Braun et al. 2017). 


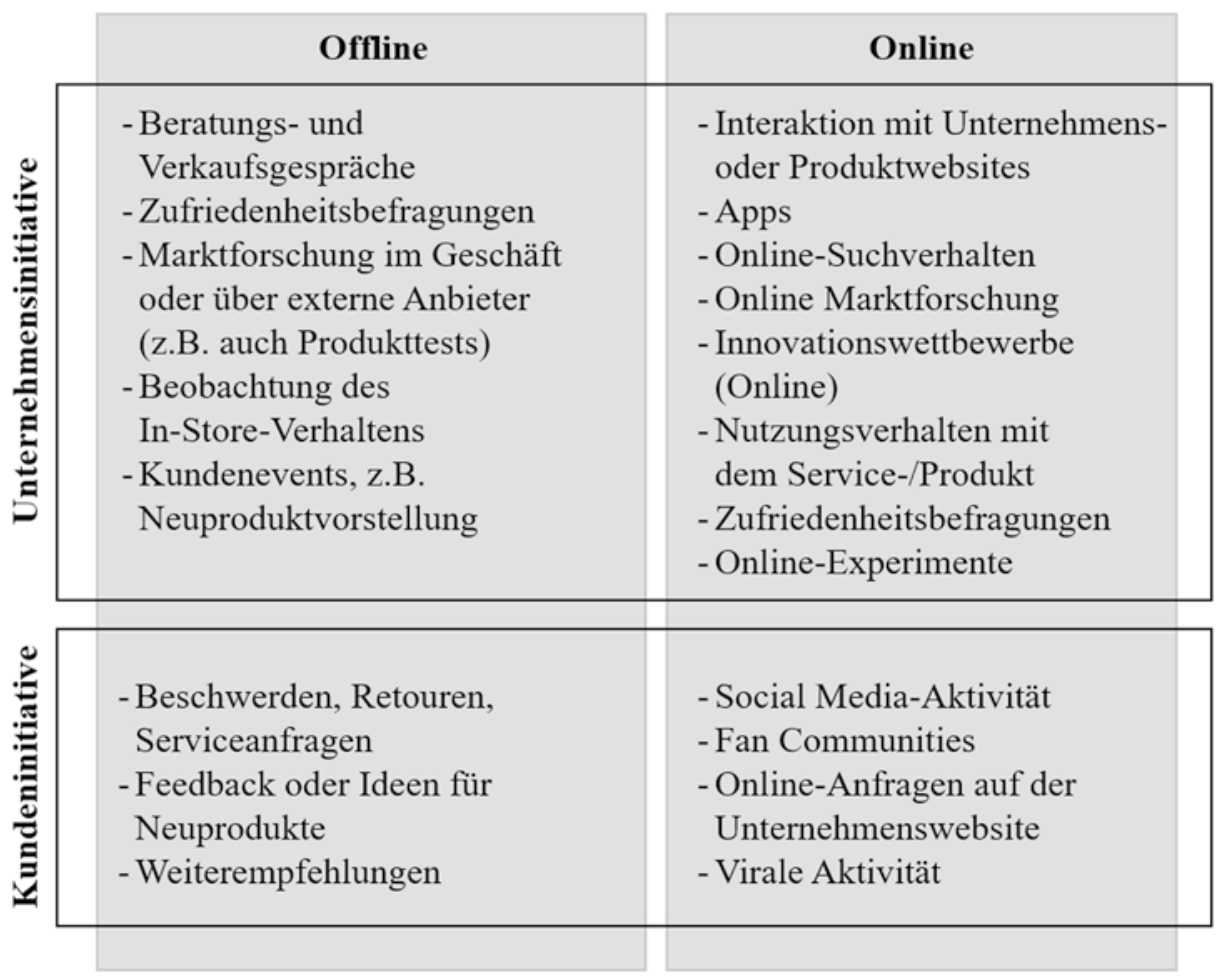

Abb. 22.3 Darstellung exemplarischer Bezugsquellen von Kundeninformationen im Online- und Offline-Umfeld. (Quelle: eigene Darstellung)

Vor dem Hintergrund diverser Bezugsquellen ergeben sich zahlreiche Informationen zu einzelnen Kunden sowie Kundengruppen, die für die Leistungsgestaltung genutzt werden können. Tab. 22.1 fasst einige Beispiele für die verfügbaren Datenpunkte zusammen:

Unabhängig davon, dass diverse und zahlreiche Kundendaten ein konkreteres Profil zeigen und somit besser für die Leistungsgestaltung geeignet sind, ist in Anbetracht der Kundeninteressen und des Datenschutzes eine Erhebung und -anreicherung dieser Daten nicht immer möglich (Rygielski et al. 2002). Neben rechtlichen Bedingungen wie der DSGVO (2016) gilt es sicherzustellen, dass sich der Kunde nie vom Unternehmen beobachtet oder seiner Privatsphäre beraubt fühlt, da dies enorme negative Konsequenzen für die Entwicklung einer vertrauensvollen Kundenbeziehung haben kann.

\subsubsection{Datenanreicherung}

Um holistische Kundenprofile erstellen und hinsichtlich relevanter Interaktionsangebote schärfen zu können, ist die Integration verschiedener Datenquellen und -typen von besonderer Bedeutung. Hierbei setzen viele Unternehmen nicht nur auf die Integration eigener 
Tab. 22.1 Exemplarische Auflistung verfügbarer Kundeninformationen. (Quelle: eigene Darstellung)

\begin{tabular}{|c|c|}
\hline \multicolumn{2}{|c|}{ Auszug möglicher Kundendaten } \\
\hline Kategorie & Beispiele \\
\hline Stammdaten & $\begin{array}{l}\text { Name, Vorname, Geschlecht, Adresse (Rechnungs- und Lieferadressen), } \\
\text { Telefonnummern, Alter, Haushaltsgröße, Beruf, Ausbildungsabschluss, } \\
\text { Zahlungsarten \& -daten, Beziehungen zu anderen Kunden }\end{array}$ \\
\hline Geografische Daten & $\begin{array}{l}\text { Wohngebiet, Entfernung zum nächsten Unternehmensstandort, Shop, } \\
\text { Service- oder Reparaturwerkstätte }\end{array}$ \\
\hline $\begin{array}{l}\text { Marketing- und } \\
\text { verkaufshistorische } \\
\text { Daten sowie After } \\
\text { Sales }\end{array}$ & $\begin{array}{l}\text { Online-Suchverhalten, Aktivität in sozialen Medien (z. B. Facebook oder } \\
\text { YouTube), Reaktion auf Unternehmenskampagnen, Anzahl Besuche, } \\
\text { Dauer und damit Relevanz der Unternehmenswebsites (v. a. via IP- } \\
\text { Adresse), App-Nutzungsverhalten, Browser, Device (z. B. Ipad, Laptop) } \\
\text { Form der Akquisition des Kunden, Erstkontakt, Reaktionen auf } \\
\text { unterschiedliche Kommunikationsmittel und -medien, geäußerte } \\
\text { Kundenpräferenzen und -wünsche } \\
\text { Kauffrequenz, Kaufmenge, Kaufort, Kaufzeiten, Kaufwert, Art und } \\
\text { Kombination der Leistungen, Nutzung von Sonderangeboten, } \\
\text { Zahlungsverhalten } \\
\text { Service-, Wartungs- und Reparaturanfragen, Retouren und Retourgründe, } \\
\text { Beschwerdeverhalten, Wiederkäufe, Anzahl geworbener Freunde und } \\
\text { Bekannte }\end{array}$ \\
\hline Nutzungsdaten & $\begin{array}{l}\text { Daten über die Nutzung von Services (z. B. Apps, Software etc.) oder } \\
\text { von Produkten, Anzahl Nutzer, Dauer und Nutzungshäufigkeit, Art der } \\
\text { Nutzung, z. B. differenzierte Betrachtung der Verwendung einzelner } \\
\text { Funktionen, Fehleraufkommen }\end{array}$ \\
\hline
\end{tabular}

Online- und Offline-Daten (z. B. Social Media-Daten, Informationen aus Loyalitätsprogrammen oder Kundenbefragungen), sondern auch auf den Bezug von Informationen von Drittanbietern. Durch den Einkauf externer Kundendaten (beispielsweise über sogenannte „Customer Data Marketplaces“) und auf Basis unternehmenseigener Profile können so ganzheitliche Perspektiven auf Individualkunden bzw. Segmente gebildet werden. (vgl. Braun 2017; Forbes 2018). Hierbei sind zentrale Vorgehensweisen der Datenanreicherung und anschließend der Datennutzung zu unterscheiden:

1. Anreicherung/Nutzung personenbezogener Daten: Es werden konkrete Informationen von Einzelkunden über diverse Kanäle, z. B. In-Store-Einkauf und Social Mediaoder Google-Daten miteinander in Verbindung gesetzt, um individuelle Maßnahmen entwickeln zu können, beispielsweise personalisierte Werbung zu den letzten Käufen bei Amazon, die auf diversen Websites gezeigt wird.

2. Anreicherung/Nutzung aggregierter Kundendaten: Kundendaten werden durch anonymisierte Informationen anderer Kunden eines ähnlichen Profils angereichert und dadurch zu Stereotypen gebündelt. Beispiele sind hier die Versendung von weiteren Produktempfehlungen auf Basis der eigenen Käufe sowie des Kaufverhaltens vergleichbarer Kunden (z. B. Apple iTunes, Amazon, nu3, Zalando, booking.com). 


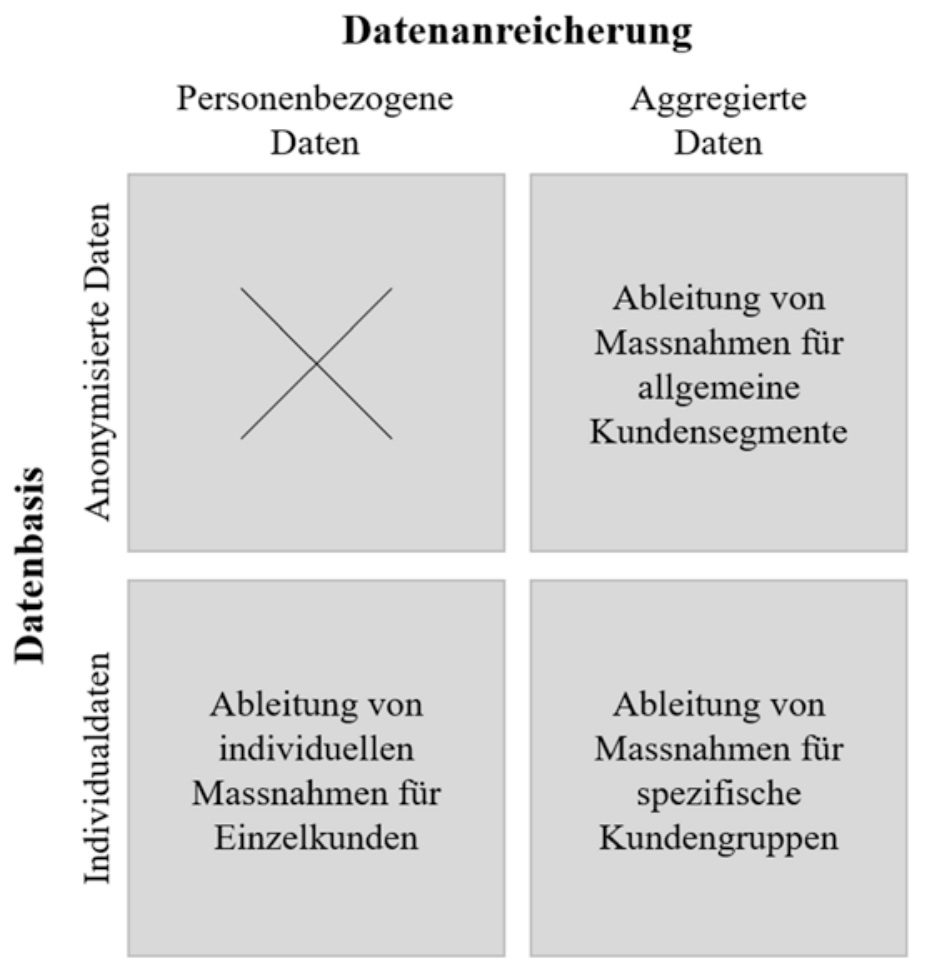

Abb. 22.4 Darstellung exemplarischer Bezugsquellen von Kundeninformationen im Online- und Offline-Umfeld. (Quelle: eigene Darstellung)

Abb. 22.4 zeigt exemplarisch die resultierenden vier Möglichkeiten der Datenanreicherung und dadurch deren Nutzung. Dabei ist zu beachten, dass im Falle anonymisierter Daten nur ein Match mit personenbezogenen Informationen möglich ist, wenn sogenannte „Linkvariablen“ für eine konkrete Zuordnung vorhanden sind. Die vier aufgezeigten Vorgehensweisen unterscheiden sich deutlich in den Potenzialen für eine individualisierte Kundenbearbeitung.

\subsubsection{Datennutzung}

Auf Basis generierter Daten bzw. umfassender Kundenprofile lassen sich viele Einsatzmöglichkeiten im Sinne der Interaktions- und Leistungsgestaltung ableiten (siehe z. B. auch Lehrer et al. 2018). Diese erstrecken sich von der Anpassung vorhandener Werbemaßnahmen bzw. der Ansprache neuer Kontakte zur Generierung neuer Leads bis hin zur Optimierung bestehender Services und Produkte. Wie bereits zuvor erläutert, sind Datenerhebung, -anreicherung und -nutzung als integrative Prozesse anzusehen. Auch nach der Implementierung entwickelter Maßnahmen bzw. Leistungen werden wieder Daten durch die Interaktion des 
Kunden mit den entsprechenden Marketingmaßnahmen, Services und Produkten generiert (z. B. Kunz et al. 2017). In der Konsequenz können diese erneut als Informationsquelle für zukünftige Leistungsentwicklungen bzw. -optimierungen genutzt werden.

\subsection{Von der Personalisierung der Ansprache bis zur Leistungsgestaltung und -optimierung}

Die zentrale Herausforderung für Unternehmen bzgl. vielfältiger Kundendaten besteht darin, eine sinnvolle Integration dieser Daten sicherzustellen und diese dann für die Leistungsgestaltung und -optimierung nutzbar $\mathrm{zu}$ machen. Folgend werden diverse Möglichkeiten der Datennutzung auf verschiedenen Ebenen der Leistungsgestaltung und -optimierung vorgestellt. Dabei wird besonderes Augenmerk auf die Potenziale der Personalisierung sowie auch der Customization (Anpassung) gelegt.

\subsubsection{Generierung neuer Leads sowie Optimierung der Ansprache bestehender Kunden}

Bei der Gestaltung von Marketingmaßnahmen zur Ansprache neuer oder bestehender Kunden können Informationen auf verschiedenen Abstraktionsgraden genutzt werden. Je mehr bzw. konkretere Informationen man über die relevanten Zielgruppen hat, desto spezifischer können Aktionen gestaltet werden. Ein Beispiel für eine erfolgreiche Umsetzung zielgruppenadäquater Ansprache ist das Unternehmen nu3, ein Anbieter für Nährstoffprodukte für die Bereiche Sport, Gesundheit und Beauty. nu3 sammelt systematisch Kundeninformationen über Interessen, Nahrungsmittelpräferenzen sowie Kaufverhalten. Dabei werden online (z. B. Interaktion auf der Website) sowie offline (z. B. telefonische Ernährungsberatung für Sportler) generierte Daten miteinander in Beziehung gesetzt und anschließend als Grundlage für die Gestaltung von personalisierten Newslettern, Produktempfehlungen sowie Anpassungen der Websiteinhalte verwendet.

\subsubsection{Interaktionsgestaltung und -optimierung}

Der Einbezug von Kundendaten bietet viele Möglichkeiten in der Gestaltung von Interaktionen an konkreten Touchpoints des Kunden mit dem Unternehmen. Informationen aktueller Kundenbedürfnisse, Interessen und Verhaltensweisen können genutzt werden, um konkrete Produkte zu empfehlen, das Sortiment und Produktportfolio anzupassen, Beratungsoptionen zu verändern oder das Verkaufspersonal zu schulen. So optimiert z. B. Harrods die eigene Werbung im Geschäft unter Berücksichtigung soziodemographischer Kundendaten, welche per Video in Echtzeit erhoben und für die gezeigten Produktempfehlungen auf den Screens ausgewertet werden. 
Ein anderer spannender Case aus dem Business-to-Business-Umfeld ist CLAAS. Der Landmaschinenhersteller nutzt nicht nur Kunden-, sondern auch sensorbasierte Produktdaten, um die Beratungs- und Verkaufsaktivitäten auf jene Kunden auszurichten, die in der Vergangenheit mit Wartungen, Reparaturen oder vermehrten Fehlermeldungen konfrontiert wurden und dadurch ein eingeschränkteres Produkterlebnis hatten.

Ein ähnliches Beispiel zum Thema Nutzung von Kundendaten zur Serviceoptimierung lässt sich bei Swiss International Air Lines finden. Kunden, die zum HON-Circle gehören oder zuvor First Class geflogen sind, werden auf Basis der erhobenen Information zu persönlichen Präferenzen (wie z. B. Essensvorlieben) und Routinen individualisiert bedient.

Auch für digitale Services lassen sich viele Möglichkeiten der datengestützten Personalisierung von Leistungen ausmachen. So identifiziert BMW im Rahmen des BMW Connected Services vorherige Fahrtmuster (Zeiten, wiederkehrende Fahrten usw.) sowie Routen zu Heim- und Arbeitswegen, die Grundlage für zusätzliche Optionen bieten. Kunden erleben so, dass z. B. beim Einstieg ins Auto die schnellste Route zur Arbeit ermittelt und via Google oder Apple Maps als direkte Push-Nachricht angezeigt wird. Darüber hinaus bietet die App Funktionalitäten im Bereich Predictive Maintenance unter Berücksichtigung des individuellen Fahrverhaltens. Ebenfalls aus dem Business-to-Consumer-Bereich verwendet auch Apple iTunes systematisch Daten über das Kundenverhalten, um das Nutzungserlebnis durch personalisierte Empfehlungen zu verbessern.

\subsubsection{Leistungsentwicklung und -optimierung}

In Bezug auf die daten- bzw. kundenbasierte Entwicklung und Optimierung von Leistungen lassen sich grundsätzlich zwei Vorgehensweisen unterscheiden, die häufig auch in der Kombination anzutreffen sind. Dabei können sich Unternehmen im Sinne einer Personalisierung auf vorhandene Kundeninformationen stützen und die Produktentwicklung bzw. -anpassung selbstständig vollziehen. Darüber hinaus gibt es die Möglichkeit, dass Kunden in den Prozess gemäß eines Customizings bis hin zur Kundenintegration in der Entwicklung von Neuleistungen einbezogen werden. Für viele Unternehmen gehört so nicht nur eine Berücksichtigung von Kundeninformationen, sondern auch die direkte Einbindung des Kunden in verschiedenen Stufen des Wertschöpfungsprozesses zum Alltag (Kleinaltenkamp et al. 1997).

In der Unternehmenspraxis werden Kunden in alle Phasen der Leistungsentwicklung, beginnend mit der Ideengenerierung, der Entwicklung erster Prototypen bis hin zur finalen Produkttestung und -optimierung einbezogen (z. B. Hofbauer 2013). Ein solches Vorgehen ist mit vielen Vorteilen verbunden: Mithilfe konkreter Kundenideen und des Konsumentenfeedbacks können neue Produkte und Services zielgruppengerecht entwickelt, erweitert oder optimiert werden, sodass mögliche Diskrepanzen zwischen Kunden- und Unternehmensperspektive schon während des Innovationsprozesses aufgedeckt werden. In der Konsequenz bietet eine frühzeitige Kundenintegration die Möglichkeit, Innovationszyklen zu verkürzen und dadurch Ressourcen zu sparen (z. B. Kleinaltenkamp et al. 1997). Die 
Intensität der Einbindung von Kunden kann variieren. Folgende drei Stufen lassen sich hier unterscheiden (z. B. Hofbauer 2013; Reichhart 2002):

1. Passive Mitwirkung: Kunden können Feedback geben, haben aber keinen Einfluss auf die Einbindung des Feedbacks in die Entwicklung von Unternehmensleistungen.

2. Aktive Mitwirkung: Kunden können zum Teil gemeinsam mit dem Produzenten neue Leistungen entwickeln und sich aktiv einbringen.

3. Aktive Partizipation: Kunden übernehmen ganze Teilaufgaben in der Leistungsentwicklung (z. B. Ideengenerierung oder Prototypenentwicklung) und tragen so einen maßgeblichen Teil zum Ergebnis der Leistungsentwicklung bei.

Als Beispiele für die Nutzung von Kundendaten und -feedback für die Leistungsentwicklung und -optimierung in der Unternehmenspraxis sind Nike, Whirlpool und SAP zu nennen. So können Kunden im Rahmen von NIKE BY YOU selbst Sportschuhe in Form, Muster und Farbe personalisieren, die dann exklusiv hergestellt werden. In diesem Bezug werden einerseits Kunden in den Herstellungsprozess miteinbezogen, aber auch andererseits durch die Offenlegung ihrer Präferenzen als Informationsquelle für zukünftige Erweiterungen des Standardsortiments genutzt.

Ein anderes Beispiel zur Nutzung von Kundendaten für die Neuproduktentwicklung ist Whirlpool als Hersteller für Heimgeräte. Whirlpool integriert sensorgenerierte Informationen zur Produktinteraktion mit user-generieten Inhalten von Social Media- und weiteren CRM-Daten, um Erkenntnisse hinsichtlich der Kundenpräferenzen und des Nutzungsverhaltens zu generieren.

Diese Datenmenge ist anschließend Grundlage für die Optimierung bestehender Produkte sowie die Leistungsentwicklung (siehe auch Woerner und Wicom 2015). Kundenfeedback erfolgreich zu erheben und in der Neuproduktentwicklung zu berücksichtigen, ist auch im Business-to-Business-Bereich üblich. So bietet SAP diverse Möglichkeiten, dass Kunden nicht nur Feedback zur bestehenden Software geben können, sondern auch direkt in sogenannten „Co-Innovationsprojekten“ gemeinsam mit dem Unternehmen neue Softwarelösungen innovieren.

\subsection{Managementimplikationen zur Umsetzung einer datenbasierten Leistungsentwicklung und -optimierung}

Möchte man sich der konkreten Konzeptentwicklung und Umsetzung einer kundendatenbasierten Leistungsgestaltung widmen, ist für Entscheidungsträger eine umfassende und zielgerichtete Analyse des Status quo unerlässlich. Dabei sind nicht nur die eigene Aktivität, sondern auch die des Wettbewerbs zu betrachten. Auf dieser Grundlage ergibt sich eine Einschätzung der bestehenden Kompetenzen sowie möglicher Marktopportunitäten. Um letztere ausschöpfen zu können, müssen die Voraussetzungen zur Implementierung im Unternehmen geklärt werden. Neben der bestehenden Infrastruktur (in diesem Zusam- 
menhang v. a. die IT-Infrastruktur sowie die Datengrundlagen), sind auch vorhandene Personalressourcen und Kompetenzen Gegenstand der Analyse.

Um letztlich den Erfolg bestehender und zukünftiger Maßnahmen im Sinne von Effektivität und Effizienz messen zu können, ist die Festlegung eines systematischen Monitorings- und Optimierungsprozesses unerlässlich. Dabei ist eine umfassende Abschätzung der Kosten-Nutzen-Relation sowie der Wirksamkeit nur dann möglich, wenn nicht nur die Performance von Einzelmaßnahmen, sondern auch die der Alternativen berücksichtigt wird.

Gegliedert in vier aufeinanderfolgende Phasen soll die folgende Checkliste in Tab. 22.2 Hilfestellungen zur Identifikation möglicher Ansätze zur Strategieentwicklung bieten.

Tab. 22.2 Managementchecklist zur Umsetzung einer datenbasierten Leistungsentwicklung und -optimierung im Unternehmen. (Quelle: eigene Darstellung)

\begin{tabular}{l|l}
\hline A) Analyse des Status quo - datenbasierte Leistungsgestaltung und -optimierung \\
\hline Interne Analyse & Inwieweit nutzen Sie derzeit Kundendaten zur \\
& Leistungsentwicklung und -optimierung (Services und/oder \\
& Produkte)? \\
Welche Resonanz erhalten Sie auf diese Maßnahmen im Vergleich \\
zum Standardangebot? \\
& Welche Aussagen können Sie hier über das Kundenfeedback oder \\
& die Nutzung treffen? Wie gestalten sich derzeit die entsprechenden \\
& KPIs von Standardmaßnahmen bzw. datenbasierten Handlungen \\
& (z. B. Conversion Rate)? \\
\hline Externe Analyse & Betrachten Sie Ihren Wettbewerb: Welche Maßnahmen verfolgen \\
& derzeit Ihre Marktmitbewerber im Sinne einer kundenzentrierten \\
& Leistungsgestaltung und -optimierung? \\
& Wo differenzieren Sie sich derzeit in entsprechenden Aktionen? \\
& Wo sehen Sie Marktpotenziale und Möglichkeiten, um sich von \\
& Ihren Wettbewerbern durch ein datengetriebenes bzw. \\
& kundenzentriertes Vorgehen zu differenzieren? \\
& Gibt es Möglichkeiten für Kooperationen (z. B. im Bereich des \\
& Datenaustauschs) in Ihrem Umfeld, um die eigene Datenbasis zu \\
& vergrössern bzw. Ihr Angebot anzureichern?
\end{tabular}

B) Analyse zukünftiger, möglicher und relevanter Maßnahmen (kurz- sowie langfristig)

Welche, der bestehenden Tätigkeiten bieten vor dem Hintergrund von Marktresonanz und Wettbewerb Erweiterungspotenziale?

In welchem Bereich der Customer Journey sehen Sie neue Anknüpfungspunkte?

Welche Möglichkeiten könnten für die aktuell wirtschaftlich interessanten Kundengruppen Ihres Unternehmens relevant sein (z. B. zur Steigerung der Raten von Wiederkauf, Cross-/UpSelling, Weiterempfehlung etc.)?

Welche zusätzlichen Möglichkeiten in diesem Bereich würden bestehende Kunden Ihres Unternehmens gerne nutzen?

Gibt es noch nicht bediente Zielgruppen, die Sie durch die Ausgestaltung entsprechender Maßnahmen gewinnen könnten? 
Tab. 22.2 (Fortsetzung)

C) Analyse der unternehmenseigenen Infrastruktur und Kompetenzen zur Umsetzung entsprechender Maßnahmen

Wie könnten sich zusätzliche Tätigkeiten im Bereich der datenbasierten Interaktions- und Leistungsgestaltung kurz- oder langfristig, z. B. innerhalb von ein bis zwei Jahren realisieren lassen?

Welche Kundendaten sind aktuell vorhanden, um bestehende
Maßnahmen zu erweitern?
Welche Daten müssten zukünftig von Kunden erhoben bzw.
verarbeitet werden, um bestehende Aktionen implementieren zu
können?
Wie können die technischen Mittel in den Bereichen der
Datenerhebung, -speicherung, -verarbeitung und -auswertung der
Informationen bewertet werden?
Wie ist generell die Qualität der IT-Infrastruktur vor dem
Hintergrund der Analyse komplexer Datenmengen in Echtzeit zu
bewerten?
Welche unternehmenseigenen Kompetenzen im Bereich der
datenbasierten Leistungsgestaltung liegen heute schon im
Unternehmen vor (v. a. Maßnahmenplanung, -implementierung,
-koordination und -pflege)?
Inwieweit können bestehende personelle Ressourcen zusätzliche
Handlungen vorantreiben bzw. ist es notwendig, dass zusätzliches
Personal akquiriert werden muss?

D) Definition des Monitorings- und Optimierungsprozesses

Welche konkreten Ziele sollen mit der betrachteten Aktivität verfolgt werden?

Welche KPIs können zur Bestimmung der Zielerreichung durch die Maßnahmen herangezogen werden?

Wie sehen mögliche Vergleichsgruppen aus? Welche

Standardprozesse bzw. Leistungen können für den Vergleich der

Effektivität und Effizienz von einem datenbasierten Vorgehen herangezogen werden?

Wie muss der Prozess gestaltet sein, dass aus den Ergebnissen der

Erfolgsschätzung eine Optimierung der betrachteten Maßnahmen erfolgen kann?

Welche Abteilung/Funktion ist für die Erfolgskontrolle und

Optimierung der Maßnahmen zuständig bzw. weisungsbefugt,

Anlass für zukünftige Maßnahmen zu geben?

\subsection{Schussfolgerung und Ausblick}

Ein systematisches Management von Kundendaten sowie die gezielte Erhebung und Nutzung von Kundenfeedback bieten Unternehmen nicht nur die Möglichkeit, die Effektivität und Effizienz der Kundenansprache, sondern auch der Leistungsgestaltung zu steigern. Voraussetzung für diese Potenzialausschöpfung ist die Sicherstellung einer quantitativ und 
qualitativ hochwertigen Datenbasis, die adäquat und ganzheitlich Kundenbedürfnisse und -verhalten abbildet. In diesem Zusammenhang bietet der vorliegende Beitrag eine Übersicht der Ausgangsbedingungen, Möglichkeiten und Handlungsempfehlungen zur Datennutzung zur Leistungsentwicklung und -optimierung.

Technologische Möglichkeiten, v. a. durch Internet of Things, Artificial Intelligence und die zunehmende Prozessautomatisierung, bieten Unternehmen in Zukunft diverse Potenziale der Datenanreicherung und der Schärfung von Kundenprofilen, bei gleichzeitig geringerem Ressourcenaufwand. Vor diesem Hintergrund müssen Entscheidungsträger bei der Datenerhebung und -nutzung neben den rechtlichen Fragestellungen auch mögliche Konsequenzen für die Beziehung zwischen Kunden und Unternehmen reflektieren, um diese nicht zu gefährden (Abb. 22.5).

\section{Literatur}

Bliemel F, Fassott G, Theobald A (2013) Electronic Commerce: Herausforderungen - Anwendungen - Perspektiven. Heidelberg

Bliemel F, Fassot G, Theobald A (2000) Electronic commerce: Herausforderungen - Anwendungen - Perspektiven. Springer Gabler, Wiesbaden

Braun L (2017) Marketing trends 2017. Personalization. Aus: http://marketing2017.ch/beispiel-seite/ personalization/. Zugegriffen am 18.10.2018

Braun L, Reinecke S, Tomczak T (2017) Kundenbindung durch Loyalitätsprogramme. In: Bruhn M, Homburg C (Hrsg) Handbuch Kundenbindungsmanagement. Springer, Heidelberg

Bughin J, Chui M, Manyika J (2010) Clouds, big data, and smart assets: ten tech-enabled business trends to watch. McKinsey Quarterly 56(1):75-86

DSGVO (2016) Europäische Datenschutz-Grundverordnung, Verordnung (EU) 2016/679 v. 27.04.2016 zum Schutz natürlicher Personen bei der Verarbeitung personenbezogener Daten, zum freien Datenverkehr und zur Aufhebung der Richtlinie 95/46/EG, ABl. Nr. L 119, S 1

Dorotic M, Bijmolt THA, Verhoef PC (2012) Loyalty programmes: current knowledge and research directions. J Manag Rev 14(3):217-237

Forbes (2018) The rise of the customer data platform and what it means to businesses. Aus: https:// www.forbes.com/sites/insights-treasuredata/2018/06/20/the-rise-of-the-customer-data-platformand-what-it-means-to-businesses/\#60aea8c053a1. Zugegriffen am 18.10.2018

Hofbauer G (2013) Customer Integration Prinzipien der Kundenintegration zur Entwicklung neuer Produkte. Working Paper. Ingolstadt

Kleinaltenkamp M, Ehret M, Fließ S (1997) Customer integration in business-to-business-marketing. In: Advances in services marketing. Deutscher Universitätsverlag, Wiesbaden, S 27-48

Kunz W, Aksoy L, Bart Y, Heinonen K, Kabadayi S, Ordenes FV, Theodoulidis B (2017) Customer engagement in a big data world. J Serv Mark 31(2):161-171

Lehrer C, Wieneke A, vom Brocke J, Jung R, Seidel S (2018) How big data analytics enables service innovation: materiality, affordance, and the individualization of service. J Manag Inf Syst 35(2):424-460

Rammstedt B (2010) Reliabilität, Validität, Objektivität. In: Wolf C, Best H (Hrsg) Handbuch der sozialwissenschaftlichen Datenanalyse. Springer, Wiesbaden, S 239-258

Reichhart S (2002) Kundenorientierung im Innovationsprozess. Deutscher Universitätsverlag, Wiesbaden 
Reinecke VS, Wortmann C (2018) Google knows it better? In: Praxis der Sinus-Milieus®. Springer VS, Wiesbaden, S 193-207

Rost J (1996) Lehrbuch Testtheorie - Testkonstruktion. Huber, Bern

Rust RT, Verhoef PC (2005) Optimizing the marketing interventions mix in intermediate-term CRM. Mark Sci 24(3):477-489

Rygielski C, Wang JC, Yen DC (2002) Data mining techniques for customer relationship management. Technol Soc 24(4):483-502

Woerner SL, Wixom BH (2015) Big data: Extending the business strategy toolbox. J Inf Technol 30(1):60-62

Wolf C, Best H (2010) Handbuch der sozialwissenschaftlichen Datenanalyse. Springer Gabler, Wiesbaden

Dr. des. Laura Braun ist wissenschaftliche Mitarbeiterin am Institut für Marketing an der Universität St. Gallen (HSG) und bei SAP Schweiz AG. In ihren Tätigkeiten beschäftigt sie sich mit den Themen CRM, Innovationsmanagement, Geschäftsmodellentwicklung und Managemententscheidungsverhalten.

Besonderer Fokus ihrer Arbeit liegt auf der erfolgreichen Integration von (externen) Stakeholdern in unternehmenseigene Innovationsprozesse. Neben Beratungsprojekten, vor allem in den Bereichen CRM und digitale Transformation, weist Frau Braun mehrere Jahre Erfahrung in der Leitung diverser Weiterbildungen für Marketingmanagement auf.

Prof. Dr. Sven Reinecke ist geschäftsführender Direktor des Instituts für Marketing an der Universität St. Gallen (HSG). Seine Forschungs- und Anwendungsschwerpunkte sind strategisches Marketing und CRM, Marketingcontrolling und Managemententscheidungsverhalten. Insbesondere beschäftigt er sich seit Jahren mit Entwicklungen des (digitalen) Kundenbeziehungsmanagements. Ferner leitet er das Exzellenzprogramm „Best Practice in Marketing“ in Kooperation mit führenden Marketingunternehmen wie Bühler, Continental Reifen, Covestro, Hansgrohe, Geberit, Miele, Swiss Life und UBS Schweiz. 
Aus:

Martin Stadelmann - Mario Pufahl

David D. Laux

Hrsg.

CRM goes digital

Digitale Kundenschnittstellen in

Marketing, Vertrieb und Service exzellent gestalten und nutzen

Springer Gabler

Wiesbaden 2020. 